\title{
The cost of evolved constitutive lac gene expression is usually, but not always, maintained during evolution of generalist populations
}

\author{
Kelly N. Phillips ${ }^{1}$ | Tim F. Cooper ${ }^{1,2}$ (D)
}

${ }^{1}$ Department of Biology and Biochemistry, University of Houston, Houston, Texas, USA

${ }^{2}$ School of Natural and Computational Sciences, Massey University, Auckland, New Zealand

\section{Correspondence}

Tim F. Cooper, Department of Biology and Biochemistry, University of Houston, Houston, TX 77204-5001, USA.

Email: t.cooper@massey.ac.nz

Funding information

Royal Society of New Zealand, Grant/Award Number: 19-MAU-082; Directorate for Biological Sciences, Grant/Award Number: DEB-1253650

\begin{abstract}
Beneficial mutations can become costly following an environmental change. Compensatory mutations can relieve these costs, while not affecting the selected function, so that the benefits are retained if the environment shifts back to be similar to the one in which the beneficial mutation was originally selected. Compensatory mutations have been extensively studied in the context of antibiotic resistance, responses to specific genetic perturbations, and in the determination of interacting gene network components. Few studies have focused on the role of compensatory mutations during more general adaptation, especially as the result of selection in fluctuating environments where adaptations to different environment components may often involve trade-offs. We examine whether costs of a mutation in lacl, which deregulated the expression of the lac operon in evolving populations of Escherichia coli bacteria, were compensated. This mutation occurred in multiple replicate populations selected in environments that fluctuated between growth on lactose, where the mutation was beneficial, and on glucose, where it was deleterious. We found that compensation for the cost of the lacl mutation was rare, but, when it did occur, it did not negatively affect the selected benefit. Compensation was not more likely to occur in a particular evolution environment. Compensation has the potential to remove pleiotropic costs of adaptation, but its rarity indicates that the circumstances to bring about the phenomenon may be peculiar to each individual or impeded by other selected mutations.
\end{abstract}

KEYWORDS

adaptation, compensation, experimental evolution, gene regulation

\section{1 | INTRODUCTION}

Fluctuating environments pose several challenges to evolving populations. While some potential adaptations might confer benefits across all relevant selection regimes (Buckling et al., 2000, 2007;
Kassen \& Bell, 1998; Satterwhite \& Cooper, 2015), others will confer benefits in some and costs in others (Bailey \& Kassen, 2012; Jasmin \& Kassen, 2007; Lee \& Marx, 2012; McGee et al., 2015; Roemhild et al., 2015). Indeed, even if unconditionally beneficial mutations are initially available, they are likely to become less common over time

This is an open access article under the terms of the Creative Commons Attribution License, which permits use, distribution and reproduction in any medium, 
(Martin \& Lenormand, 2015; Satterwhite \& Cooper, 2015; Schick et al., 2015). When a mutation that confers a benefit in one environment, and a cost in another, fixes in a population, it creates selection for subsequent mutations that compensate for that cost (MaisnierPatin et al., 2002; Moore et al., 2000; Moura de Sousa et al., 2017; Wood et al., 2013).

Compensatory mutations have long been used in molecular genetic studies as a tool to identify physical and genetic changes that can suppress the effects of a focal mutation, thereby identifying interacting components (Blank et al., 2014; Jarvik \& Botstein, 1975; Kacar et al., 2017; Manson, 2000; Ponmani \& Munavar, 2014; van Leeuwen et al., 2016). Increasingly, they are also recognized as being important in broadening the scope of evolutionary trajectories a population can follow (Szamecz et al., 2014; Zee et al., 2014), allowing adaptations to be selected that might otherwise prove to be evolutionary dead-ends (Covert et al., 2013; Harrison et al., 2015), and influencing the ability of populations to simultaneously adapt to multiple environments (Melnyk et al., 2017).

Mutation interactions arising following selection in fluctuating environments have been extensively studied in the context of the evolution of antibiotic resistance. Antibiotic resistance mutations often confer a cost to bacteria in antibiotic-free environments (Moura de Sousa et al., 2017; Nilsson et al., 2003; Rozen et al., 2007). This cost generates selection for subsequent compensatory mutations that relieve the cost (Björkman et al., 1998, 2000; Levin et al., 2000; Nagaev et al., 2001). Compensation allows resistance mutations to be maintained when they would normally be selected against, influencing short-term evolutionary outcomes and perhaps longer-term potential. Similar patterns of compensatory mutations depending on earlier resistance mutations for their benefit have been seen in studies of bacterial resistance to bacteriophage (Lenski, 1988; Wielgoss et al., 2016). Compensation during evolution has also been found to occur to overcome the loss of essential genes (Blank et al., 2014), the negative effects of synonymous (Knöppel et al., 2016), and gene deletion (Szamecz et al., 2014) mutations and to restore a social trait (Zee et al., 2014).

In contrast to studies that have focused on mutations that compensate for a specific genetic perturbation, few studies have examined compensation during more general adaptation to an environment, especially when this adaptation involves repeating rounds of selection in contrasting environments. This distinction might be important. Compensation to a specific genetic perturbation, such as deletion of a focal gene, is thought to generally act locally (Brandis et al., 2012; Filteau et al., 2015; Szamecz et al., 2014), although it can also arise in pathways unrelated to the perturbation (Blank et al., 2014). At least in experimentally evolving populations, adaptation often involves mutations in regulatory genes that are likely to have highly pleiotropic consequences (Cooper et al., 2003; Kurlandzka et al., 1991; MacLean et al., 2004; Rosenzweig et al., 1994). If costs of such pleiotropic mutations are revealed following an environmental change, it is not clear how subsequent compensatory mutations might affect fitness in the original environment. Indeed, it is easy to imagine that compensation causing a reduction in the cost of a focal mutation in a new environment might be associated with a reduction of the original benefit. In that case, reversion to the original environment might select for reversal of the effects of the compensatory mutation. This could occur through its direct reversion or through a second compensatory mutation, creating potentially complex patterns of environmentally dependent epistatic interactions between selected mutations.

The particular nature of the environmental fluctuations a population is exposed to is expected to play a major role in the selection of compensatory mutations. In a rapidly changing environment, mutations that increase in frequency are likely to confer a net benefit across the different environments (Buckling et al., 2007; Melnyk et al., 2017; Turner \& Elena, 2000). With this limitation, such mutations can only confer at most relatively small costs in any one environmental component so that the strength of selection for compensation may be small (Poon \& Chao, 2005, 2006). In a more slowly fluctuating environment, mutations selected in one component might fix before the population experiences a second component in which they might confer substantial costs (Bennett \& Lenski, 2007; Kassen \& Bell, 1998; Phillips et al., 2016). Such differential costs are consistent with the generally higher between-environment tradeoffs seen in populations selected slowly compared to quickly fluctuating environments (Bono et al., 2017; Satterwhite \& Cooper, 2015; Schick et al., 2015).

We examine compensation to an adaptive mutation selected in a series of experimentally evolved populations selected in environments that contained either lactose or glucose alone or a combination of lactose and glucose fluctuating daily or every 2,000 generations (Cooper \& Lenski, 2010). Mutational inactivation of the Lacl repressor was rapidly selected in many of the replicate bacterial populations that were selected in the presence of lactose (Quan et al., 2012). Loss of Lacl causes the lac operon, a set of genes required for utilization of lactose, to be constitutively expressed (Markiewicz et al., 1994; Quan et al., 2012). When engineered into the ancestor of the evolution experiment, constitutive expression of the lac operon provided a benefit of $~ 9 \%$ during growth in lactose by shortening the lag time before resumption of growth following transfer into fresh medium (Quan et al., 2012). It also conferred a cost of $\sim 3 \%$ in an environment containing glucose as the sole resource, probably due to some combination of the energetic cost of expressing unnecessary genes and toxicity of the LacY permease (Dekel \& Alon, 2005; Eames \& Kortemme, 2012; Quan et al., 2012; Stoebel et al., 2008). In populations selected in environments containing both lactose and glucose, this trade-off in the effect of laclmutations creates potential for subsequent mutations to provide a fitness benefit by compensating for the cost of the mutation in glucose.

We test whether evolved populations that fixed the lacl- mutation, and therefore constitutively express the lac operon, evolved mechanisms that alleviate the cost of this expression during growth in glucose and, if so, whether this compensation comes at a cost of the benefit conferred by lacl- in lactose. To do this, we isolated strains from populations evolved for 8,000 generations in lactose, 
glucose, and combinations of both fluctuating daily and every 2,000 generations. The lacl- mutation was reverted in those strains that had substituted it, and its effect on fitness was determined. We found that the fitness cost of lacl- in glucose was variable, including, in one strain, becoming beneficial, but did not differ consistently between populations evolved in lactose only, where compensation is not expected to be selected, and in environments containing glucose, where it is. Similarly, strains varied in their relationship between the fitness benefit conferred by the lacl- mutation in lactose and costs in glucose, but this variation did not depend on their selection environment. Together, these results demonstrate the potential for the action of compensatory mutations to influence costs of adaptation but indicate that their effects may either be idiosyncratic or be overwhelmed by the effects of additionally selected mutations.

\section{2 | MATERIALS AND METHODS}

\section{1 | Bacterial strains and strain construction}

Bacterial strains were selected from replicate populations started with Escherichia coli B strains REL606 and REL607, which are isogenic except for a single base change in araA, which determines the ability to utilize arabinose, and a mutation in $\operatorname{rec} D$ that also appears to be neutral (Tenaillon et al., 2016). Populations were evolved in Davis-Mingioli (DM) minimal media supplemented with different combinations of glucose $(175 \mu \mathrm{g} / \mathrm{ml})$ and lactose $(210 \mu \mathrm{g} / \mathrm{ml})$ (Cooper \& Lenski, 2010). The evolution environments included DM supplemented with lactose (Lac), daily fluctuations of glucose and lactose $(G / L)$, or long-term switching from glucose to lactose $\left(G_{-} L\right)$ or from lactose to glucose ( $L_{-} G$ ) every 2,000 generations. Each population was evolved for 8,000 generations, except for one G/L population, which needed to be restarted from an intermediate point during the evolution experiment and was evolved for 7,000 generations (G/L2). This difference has no consequence to the results reported here. Six replicate populations were evolved in each environment, and the replicate number is indicated by the number following the evolution environment designation. Clones with the lacl- mutation were isolated from populations based on their growing as a blue colony on indicator plates that contained X-gal (5-bromo-4-chloro-3-indo lyl-beta-D-galactopyranoside) and glucose (TGX plates) (Figure 1; Quan et al., 2012). On TGX plates, the blue phenotype is indicative of a strain constitutively expressing the lac operon. A total of nine clones were chosen, one from each of three lactose-only populations, a G_L population, and five G/L populations. In all clones, the lacl gene was amplified and sequenced to verify the presence of a mutant Lacl repressor that we assume to be the cause of constitutive lac operon expression (Figure 1). Amplification was carried out using the primers: 5'-GCGGAGCTGAATTACATTCC-3' (11-F) and 5'-GGGTGCCTAATGAGTGAGCT-3' (12-R).

To determine the effect of substituted lacl- mutations, we first constructed lacl+derivatives of our focal evolved strains. To do this, we PCR-amplified the ancestral allele using primers $11-\mathrm{F}$ and $12-\mathrm{R}$,

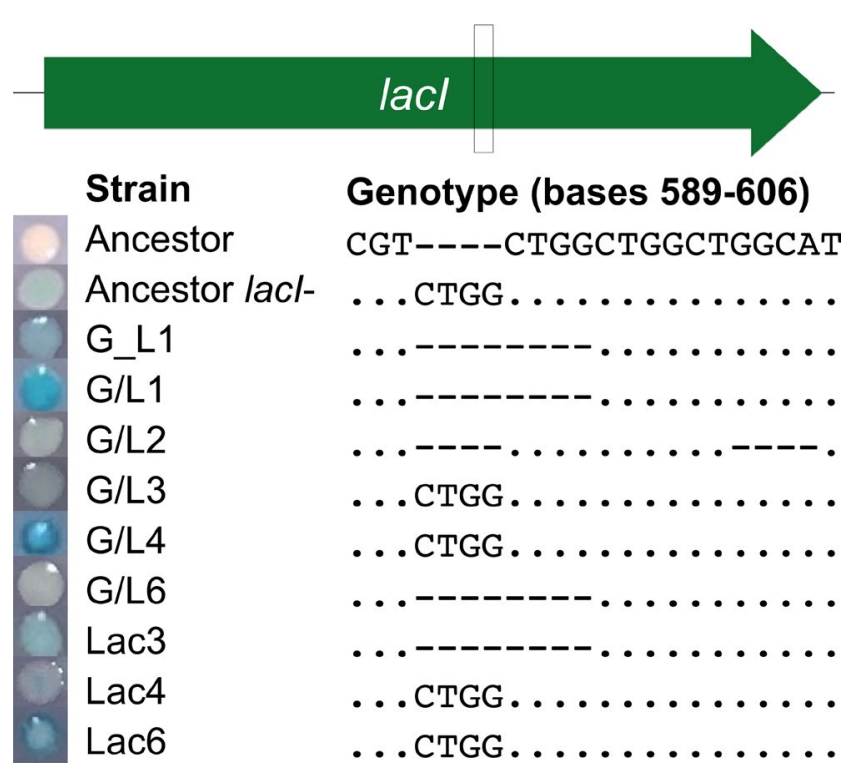

FIGURE 1 X-gal phenotype and lacl mutations in focal clones. A representative colony of each clone grown on TGX indicator plates is shown at left. All mutations across evolved clones were either a 4 bp insertion or deletion frameshift mutation in a mutational hotspot region of lacl (box at top). Only the insertion is shown for the ancestor lacl-, but the deletion was also assessed and had the same fitness effect and X-gal phenotype

ligated this product into pCR2.1 using a TA cloning kit (Invitrogen). The plasmid was used to transform TOP10F' cells, which were blue/ white screened to identify transformants having a plasmid insert. pCR2.1::lacl+plasmids were purified, and the lacl+fragment excised and cloned into pDS132 using enzymes Sacl and Xbal. The resulting plasmid, pDS132::lacl+, was used to transform MFDpir cells (Ferrières et al., 2010). MFDpir (pDS132::|acl ${ }^{+}$) was separately conjugated with each target evolved strain by mixing donor and recipient at a 1:2 ratio, respectively. Conjugation was carried out on LB agar plates supplemented with 2,6-diaminopimelic acid (30 $\mu \mathrm{M})$, which MFDpir needs for growth, for $3-4 \mathrm{hr}$ at $37^{\circ} \mathrm{C}$. The conjugation mixture was resuspended in $200 \mu \mathrm{lDM}$ medium and plated onto minimal glucose agar (MG) supplemented with chloramphenicol (Cm; $20 \mu \mathrm{g}$ / $\mathrm{ml}$ ) to select for recipient strains with the pDS132 plasmid successfully integrated into the chromosome (Philippe et al., 2004). After overnight incubation, six colonies were restreaked onto $\mathrm{MG}+\mathrm{Cm}$ agar, again incubated overnight, and then restreaked a second time to the same medium. A colony descended from each of the original six was resuspended in $500 \mu \mathrm{LM}$ base liquid media and plated onto sucrose plates supplemented with X-gal to select for excision of the pDS132 plasmid (Philippe et al., 2004) and screen for clones that had retained the introduced lacl+allele. One blue colony and one white colony were selected from each plate and restreaked twice onto sucrose $+\mathrm{X}$-gal plates. White colonies indicate clones that successfully integrated the lacl+allele. Blue colonies, which retained the original lacl-allele, were used as negative controls to test for the presence of additional mutations that occurred during the allele replacement process. All clones were tested for proper pDS132 plasmid excision 
by checking for chloramphenicol sensitivity. The lacl+insertion was verified by sequencing.

To evaluate the possibility of secondary mutations being inadvertently added during construction of lacl+strains, we measured the fitness of control strains that went through the conjugation process but did not retain the introduced lacl+allele in competition with an otherwise isogenic strain with a distinct neutral ara marker. The ara marker strains were constructed either by pairwise conjugations between the evolved strain and MFDpir (pDS132::ara-) (same method as above except X-gal was not used in the media) or by plating $100 \mu$ l of overnight LB cultures onto minimal arabinose agar (MA) and selecting for spontaneous ara+mutants. Indistinguishable fitness between competing strains was interpreted as indicating the absence of fitness-effecting secondary mutations.

\section{2 | Genome sequencing}

We report sequence of one evolved clone isolated from the G/ L4 population. Genomic DNA was isolated and purified using the Wizard Genomic DNA Purification Kit (Promega). Libraries were created following the Nextera XT DNA Library Prep Kit protocol with Nextera XT Index Kit v2 adapters (Illumina). Libraries were pooled and sequenced on an Illumina NextSeq, producing 150 base-pair, and the Breseq computational pipeline was used to align reads to the REL606 reference sequence and identify mutations (Deatherage \& Barrick, 2014).

\section{3 | Fitness assays}

Fitness effect of the lacl- mutation in each isolated strain was measured in glucose $(175 \mu \mathrm{g} / \mathrm{ml})$ and lactose $(210 \mu \mathrm{g} / \mathrm{ml})$-only environments. Cells were transferred from freezer stocks initially to LB medium and then, after overnight growth, to minimal medium supplemented with glucose or lactose as used in the particular competition assay. Strains were initially acclimated to the assay environment over two $24 \mathrm{hr}$ transfer cycles with a 1:100 dilution occurring between each cycle. Preconditioned competitors were mixed at a 1:1 ratio by diluting each competing strain 1:200 directly into the assay environment. Competitions were carried out over two (lactose competitions) or four (glucose competitions) transfer cycles. A greater number of transfer cycles was used in glucose to be able to detect the small fitness differences between strains generally present in that environment. On the initial and final day of competitions, cells were plated onto TGX plates and incubated at $37^{\circ} \mathrm{C}$ for $18-20 \mathrm{hr}$ in order to distinguish competing genotypes. Relative fitness effect of the lacl- mutation was determined based on the change in density of blue (lacl-) and white (lacl+) colonies on TGX plates using the formula: In(blue ${ }_{2} \times 100 \mathrm{t} /$ blue $\left._{0}\right) / \ln \left(\right.$ white $_{2} \times 100 \mathrm{t} /$ white $\left._{0}\right)$, where subscripts indicate the time at which competitor density was estimated, and $t$ accounts for transfer cycles during the competition
(Lenski et al., 1991). Test competitions checking for additional mutations occurring during allele replacement procedures or selection of spontaneous ara+mutants were performed as described above, except competitors were distinguished by plating onto tetrazolium arabinose agar (TA).

\section{4 | Expression assays}

Expression of the lac operon was measured using a GFP reporter construct controlled by the $\mathrm{P}_{\text {lac }}$ promoter region including the $\mathrm{O} 1$ and $\mathrm{O} 3 \mathrm{Lacl}$ operator sequences, and native primary CRP binding site (Quan et al., 2012). This reporter was previously cloned into a mini-Tn7 cassette in a suicide-vector (Quan et al., 2012) and was introduced into target strains by tri-parental conjugations between a target recipient evolved strain, a donor strain (MFDpir (pUC18R6KT:: $P_{l a c}-$ GFP, $\left.k^{r}{ }^{r}\right)$ ), and a helper strain (MFDpir (pTSN2)) (Choi et al., 2005; Ferrières et al., 2010; Quan et al., 2012). Strains were mixed at a 3:1:1 ratio (recipient: donor: helper) on LB +DAP $(30 \mu \mathrm{M})$ agar and incubated at $37^{\circ} \mathrm{C}$ for $3 \mathrm{hr}$. The conjugation mix was resuspended in DM medium and plated onto LB +kanamycin $(\mathrm{Km} ; 60 \mu \mathrm{g} / \mathrm{ml})+$ isopropyl $\beta$-D-1-thiogalactopyranoside (IPTG; $1 \mathrm{mM}$ ) agar plates. Kanamycin selects for clones that successfully obtained the $\mathrm{P}_{\text {lac }}$-GFP reporter while IPTG induces expression of the reporter allowing identification of clones that stably expressed GFP. After 24-36 hr of growth, six fluorescent colonies were restreaked onto $\mathrm{LB}+\mathrm{Km}(60 \mu \mathrm{g} / \mathrm{ml})$ plates. Restreaked colonies were streaked a second time on LB $+\mathrm{Km}+$ IPTG plates and then tested for absence of the delivery plasmid by spotting colonies on LB $+\mathrm{Ap}(100 \mu \mathrm{g} / \mathrm{ml})$ agar. Insertion of the Plac-GFP reporter into the attTn7 site was confirmed by PCR (primers: 5'-TAACAGCCAGCACCACGCCG-3' (120-F) and 5'-CGCGAATCCGATCTGGCGCT-3' (121-R)). Transposition of the reporter into each recipient strain's attTn7 site allows consistent insertion of the reporter into the same region of the genome, minimizing divergent effects on reporter fitness and expression costs.

To measure $\mathrm{P}_{\text {lac }}$-GFP reporter expression, reporter strains were grown overnight in LB broth from freezer stocks, diluted 1:10,000 in the assay environment, and allowed to grow for $24 \mathrm{hr}$. The following day cultures were diluted again 1:10,000 in the assay environment and grown in a VersaMax spectrophotometer (Molecular Dynamics, CA) until mid-log phase $\left(\mathrm{OD}_{450} \sim 0.1-0.15\right)$ to allow measurement of a steady-state level of $\mathrm{P}_{\mathrm{lac}}$-GFP reporter expression. $\mathrm{P}_{\text {lac }}$-GFP expression was measured in an Accuri C6 flow cytometer (Becton Dickinson, NJ). Assay environments included DM+175 $\mu \mathrm{g} / \mathrm{ml}$ glucose, $\mathrm{DM}+210 \mu \mathrm{g} / \mathrm{ml}$ lactose.

\section{5 | Statistical analyses}

Data were analyzed using R (version 3.5.0; R Core Team, 2018). Dunnett's tests for one against many comparisons were performed using the glht function in the multcomp package. Where appropriate, 
experimental block was included in analyses as a random effect in mixed-model ANOVA performed using the Imer function in the Ime4 package. Fixed effects were tested for significance by comparing models fitted with and without the factor of interest using a chisquare test.

\section{3 | RESULTS}

\section{1 | Evolution of compensatory mutations}

In the ancestor to our evolution experiment, mutations that inactivate the Lacl repressor, and lead to constitutive expression of the lac operon, confer a fitness cost of approximately $2.2 \%$ during growth in glucose (Quan et al., 2012). To determine whether this cost is compensated during evolution in environments containing both glucose and lactose resources, and, if so, whether this compensation differed depending on the presentation of the two resources, we reverted evolved lacl- mutations in nine clones isolated from populations selected in lactose alone (Lac, three populations), long-term switching of glucose and lactose (G_L, one population), or daily switching of glucose and lactose (G/L, five populations). We found that the cost of lacl-in glucose was significantly changed only in evolved clone G/ L4 where the mutation had become beneficial (Figure 2; Dunnett's Test, $p<.001)$. This result indicates the presence of sign epistasis, in that the lacl- mutation changed from being deleterious in the ancestor to being beneficial in the evolved background. No difference in evolved lacl- costs was detectable among other evolved clones $\left(\chi^{2}=7.82, d f=8, p=.45\right)$.

\section{2 | Effect of environment on compensatory mutations}

Although the cost of lacl-was significantly changed in only one clone when compared to the ancestor, there may be changes in costs apparent when grouping clones based on their evolution environment. To test this, we compared the effect of the lacl- mutation on fitness in glucose of each evolution environment group (strains evolved in $\mathrm{Lac}, \mathrm{G} / \mathrm{L}$, or $\left.\mathrm{G}_{-} \mathrm{L}\right)$. We expect clones evolved in the presence of fluctuations of glucose and lactose (i.e., $G / L$ and $G \_L$ selection environments) to have a reduced fitness cost of the lacl- mutation in glucose because compensation for the original cost would provide an advantage. By contrast, selection for compensatory mutations was expected to be reduced or absent in the lactose-only environment. We found no significant difference in cost of the lacl- mutation measured in the glucose environment among clones isolated from different evolution environments whether or not the outlier clone, G/L4, was included (ANOVA with G/L4: $F_{2,94}=1.68, p=.19$; without $\left.\mathrm{G} / \mathrm{L} 4: F_{2,88}=0.98, p=.38\right)$.

\section{3 | Pleiotropic effect of lacl- compensation}

Given that compensation for the cost of constitutive lac expression evidently can occur, one explanation for the low frequency at which it does occur is that it imposes a correlated cost in lactose. For example, it might be that compensation to constitutive expression of the lac operon involves a reduction in the maximum level of lac expression, perhaps reducing fitness in lactose and thereby causing

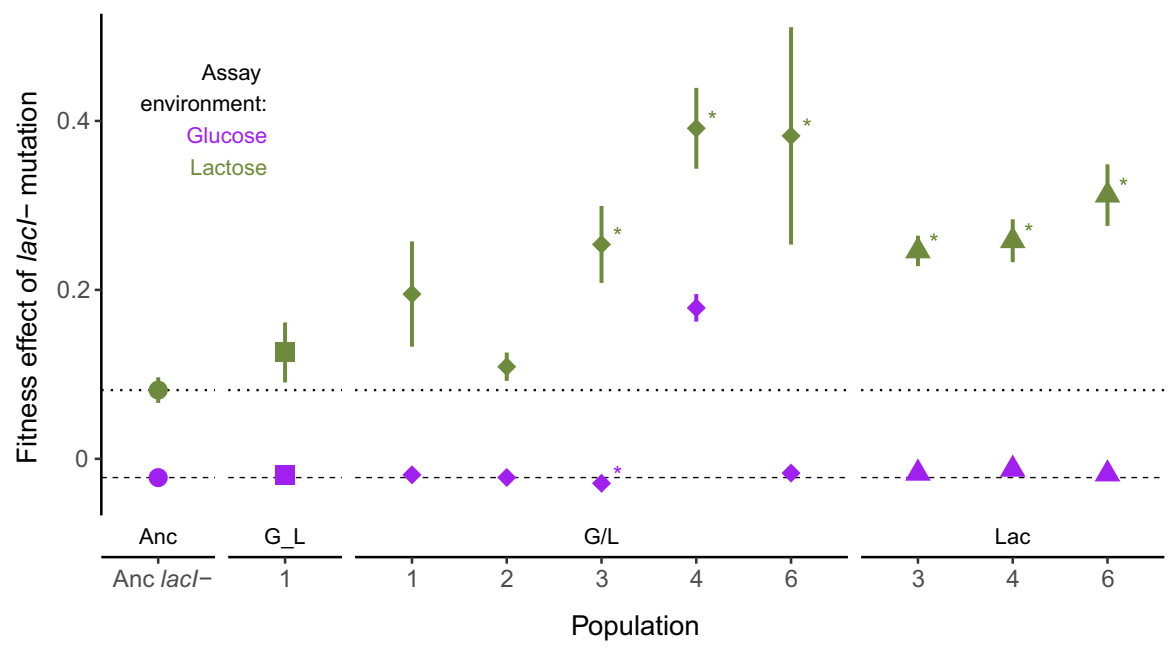

FIGURE 2 Fitness effect of lacl-in the ancestor and evolved genetic backgrounds measured in minimal glucose (purple symbols) and lactose (green symbols) environments. Asterisks indicate clones with significantly different lacl- fitness effect when compared to the effect in the ancestor (Dunnett's test, $p<.05$ ). Symbols and error bars indicate mean and standard error of at least 10 (in the glucose environment) or three (in the lactose environment) replicate fitness estimates. Note that error bars are smaller than symbols for many of the glucose fitness estimates. The dashed lines represent the fitness effect of lacl- in the ancestor in glucose (dashes) and lactose (dots). Evolution environments are shown directly above clone names which denotes the population a clone was selected from ("Anc" indicates the ancestor). In the glucose environment, one clone had a significantly higher fitness than the ancestor indicating that compensatory mutations are alleviating the cost of lacl-in glucose 
compensation to be selectively disfavored. To test this possibility, we examined the fitness effect of lacl- mutations across glucose and lactose environments. To determine if there was any trend of a lower cost of lacl- in glucose corresponding to a lower benefit in lactose, we determined the relationship between the fitness effect of lacl- mutations in glucose and lactose across all strains. We found a marginally significant positive correlation between fitness in the two environments, indicating that a low cost of the lacl- mutation in glucose was, if anything, associated with an increased benefit in lactose (Figure 3; Spearman's rank correlation, rho $=0.73, p=.021$ ). The significance of the correlation is dependent on the G/L4 evolved clone that compensated for the lacl- mutations cost in glucose. When that clone was omitted from the analysis, the correlation was no longer significant, though was still positive ( $r$ ho $=0.63, p=.08$ ). Focusing on the G/L4 clone revealed that the lacl- mutation is beneficial in glucose and its effect in lactose is significantly higher than in three of the evolved clones tested, as well as the ancestor (G_L1, G/L1, and $\mathrm{G} / \mathrm{L} 2$; Dunnett's test, $p<.05)$. Together, these results indicate that there is no trade-off with fitness in lactose that limits selection for compensation of lacl- costs in glucose.

\section{4 | Mechanisms of lowered costs}

To determine if lac operon expression is associated with changes in the fitness effects of the lacl- mutation, we measured lac operon expression in glucose using a reporter that is controlled by the promoter region, $\mathrm{P}_{\text {lac, }}$ that drives expression of the lac operon (Figure 4). Expression of the lac operon contributes to the cost of constitutive expression, so we expected a negative relationship, such that clones that had higher lac operon expression would have lower fitness in the glucose environment (i.e., a higher cost; Dekel \& Alon, 2005; Stoebel et al., 2008). In fact, there was no correlation (Figure 5a; Spearman's rank correlation, rho $=0.27, p=.49$ ). This is especially surprising because half of the evolved strains had significantly higher lac expression in glucose than the ancestor lacl-, so that an effect of lac expression on fitness could have been detected (Dunnett's test: G_L1, G/L4, Lac3, Lac4, Lac6 $p<.05)$. That increased expression was not associated with any fitness cost might indicate the action of compensation to some portion of the cost that would otherwise be associated with increased lac expression. Alternatively, there could be a limit to the cost associated with constitutive lac operon expression (Eames \& Kortemme, 2012), although the model most analogous to the situation prevailing in our experiments predicts exponentially increasing costs with increasing expression (Dekel \& Alon, 2005).

All clones except G/L4 had similar lacl- associated fitness costs when compared to the ancestor, but clones varied when it came to differences in expression compared to the lacl- ancestor. G/L4 had equal lowest lac expression in glucose (Dunnett's test, $p<.05$; except for G/L3, $p=.87$; and G/L6, $p=.43$ ), and all G/L clones had lower expression in glucose when compared to all Lac clones. Together, these results indicate that there was some differential evolution of lac expression based on environment, but that consequences do not consistently map to fitness effects: clones with similar expression levels in glucose have different lacl- fitness effects (G/L4 compared to G/L3 and G/L6), and clones with similar lacl- fitness effects have different expression (G/L compared to Lac clones). Evidently, reduced lac operon expression cannot explain all of the decreased costs in glucose and alleviation of the cost is dependent on other mutations in the evolved background.

Finally, our reporter strains allow us to address the relationship between lac expression and fitness in the lactose environment. We expected a positive correlation between lac expression and fitness if higher lac expression directly contributed to fitness in lactose. In fact, although all evolved clones had increased lac expression, changes were not correlated with fitness (Figure 5b; Spearman correlation, rho $=-0.18, p=.64$ ). Moreover, although the lacl- mutation conferred one of the biggest benefits when added to the G/L4 clone, lac expression was not significantly different in this clone compared to other evolved clones (Dunnett's test, $p>$.05). These results suggest that the benefit of higher expression depends on the genetic background in which it occurs and that most of the changes in maximum lac expression are not caused by lacl-itself but by differences in the broader genetic background.

\section{4 | DISCUSSION}

Few studies have focused on the influence of compensatory mutations during adaptation to a general environment rather than to a specific genetic perturbation. Our results demonstrate that compensation to deleterious consequences of an adaptive mutation is possible but is rare among evolved clones in this experiment. Of the nine clones tested, only one clone evolved a mechanism to alleviate the cost of constitutive lac operon expression in glucose (Figure 2). No differences in compensation were evident comparing groups of clones evolved in different environments, including some that were and some that were not expected to select for compensation.

Compensation of deleterious mutation effects has been observed in many systems and contexts-including compensation of costs due to antibiotic resistance, adaptation, and gene deletion (Björkman et al., 1998, 2000; Blank et al., 2014; Knöppel et al., 2016; Levin et al., 2000; Nagaev et al., 2001; Szamecz et al., 2014; Zee et al., 2014). A common theme is that compensation is readily selected, consistent with there being many available genetic mechanisms. For example, one study found $68 \%$ of singlegene deletion slow growth mutants could be compensated to restore growth to near wild type within $\sim 400$ generations (Szamecz et al., 2014). Indeed, compensation to the costs of the lacl- mutation apparent in glucose containing environments was possible in our experiment, but occurred in only one of the nine clones we examined. One explanation is that compensation is limited not by mutational opportunity but by selection for those mutations. Our populations evolved asexually so that beneficial mutations of large effect outcompete those of smaller effect (Gerrish \& Lenski, 1998). Thus, even if they arise, small benefit compensatory 


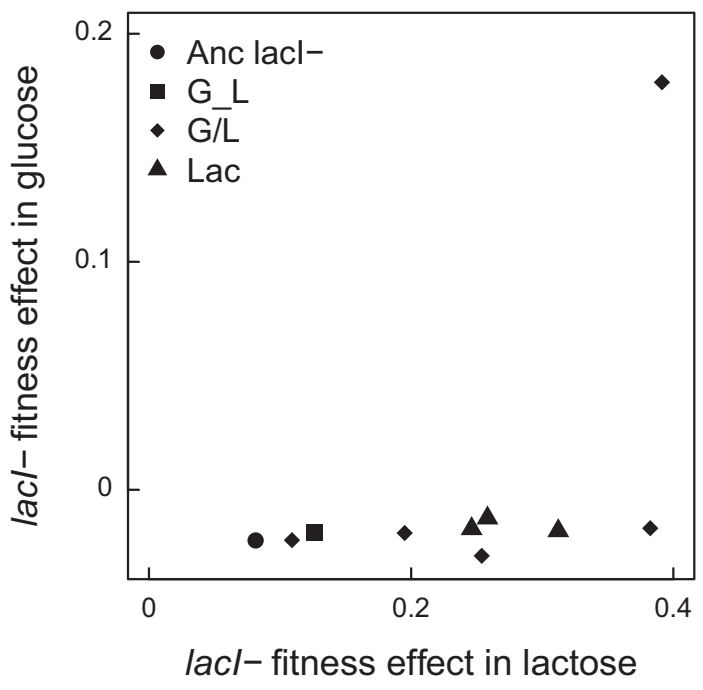

FIGURE 3 Fitness effect of lacl-in ancestor and evolved clones measured in glucose and lactose environments. Symbol shapes indicate the evolution environment or genotype of each clone. The ancestral measurements (circle) indicate the expectation if there were no epistatic interactions between lacl-and other mutations in the evolved background

mutations might be outcompeted until mutations of large effect are exhausted (Leon et al., 2018). Our populations exhibit higher fitness gains at early versus later time points (Satterwhite \& Cooper, 2015); however, the latter gains may still be larger than the small $\sim 2.2 \%$ cost of lacl- in glucose. Indeed, the G_L populations steadily increased in glucose fitness up to 6,000 generations so compensatory mutations may not be sufficiently competitive to be selected (Satterwhite \& Cooper, 2015). By contrast, G/L populations had small or undetectable fitness change across glucose and lactose environments after 4,000 generations, which would have allowed selection of small benefit mutations and may be why only a G/L clone was suggested to have compensatory mutations (Satterwhite \& Cooper, 2015).

Another possible explanation for the rarity of compensation is that multiple mutations are required to compensate the cost of laclin glucose without affecting its benefit in lactose (Poon et al., 2005). Multiple compensatory mutations will take longer to fix and will be rare because successful compensation can depend on the order in which each required mutation occurs (Gong et al., 2013), the presence/absence of other mutations in the genetic background (Lunzer et al., 2010; Shah et al., 2015), and a clone's fitness at each step relative to others in the population which can subject an intermediate clone to being purged by purifying selection (Gerrish \& Lenski, 1998). Replacing lacl- with a functional Lacl repressor in the G/L4 clone, in which compensation did occur, significantly reduces fitness in glucose and expression of the lac genes. In other words, this clone has somehow rewired the lac network such that lac operon expression is beneficial for growth in glucose. The basis of this rewiring is unknown but might depend on multiple mutations as is the case, for example, in the case of evolved citrate utilization

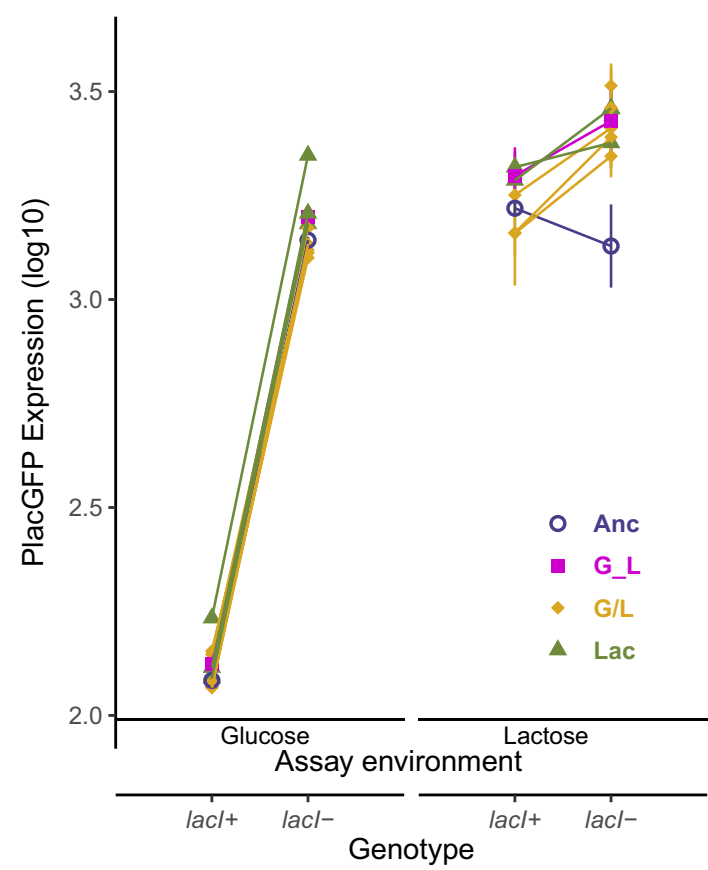

FIGURE 4 Plac-GFP reporter expression of lacl+and laclclones in glucose and lactose. The symbols and colors indicate the genetic background and evolution environment of each clone. The $\mathrm{X}$-axis shows the genotype and assay environment that Plac-GFP expression was measured in. Each measurement is the average of the median expression values of independent replicates $(n=14$ in glucose and $n=4$ in lactose). Error bars show standard error of the mean (note that error bars are generally covered by symbols in the glucose environment assays)

selected in a population started from the same ancestor as the one used here (Blount et al., 2008, 2012; Quandt et al., 2015).

We consider that LacY is the best candidate lac gene to be involved in some new compensatory interaction. Not only is it the likely source of the cost of constitutive lac expression (Eames \& Kortemme, 2012), but it can also support glucose uptake, though, as characterized, that requires a mutational change in the enzyme that did not occur in clone G/L4 (Gram \& Brooker, 1992; King \& Wilson, 1990; Sahin-Tóth et al., 2001). Although glucose transport is not likely to limit growth at the concentration used in this experiment, it is possible that an alternative import mechanism could provide an advantage by reducing dependence on the phosphotransferase system (Ferenci, 1996; Jahreis et al., 2008; Postma et al., 1993). This system is a common mutational target in populations evolved from the same ancestor and selected in a glucose environment, suggesting it is not optimized for growth in the selective environment prevailing in our experiment (Woods et al., 2006). Sequencing of a clone isolated from the $\mathrm{G} / \mathrm{L} 4$ population identified a mutated gene, sohB, that is a candidate for interacting with LacY and that was not mutated in the other populations we examined here or in lines that evolved only in glucose (Table S1). SohB is a peptidase active, along with LacY, in the cell periplasm (Baird et al., 1991). Although the function of SohB is not well characterized, it has been shown to be able to compensate 

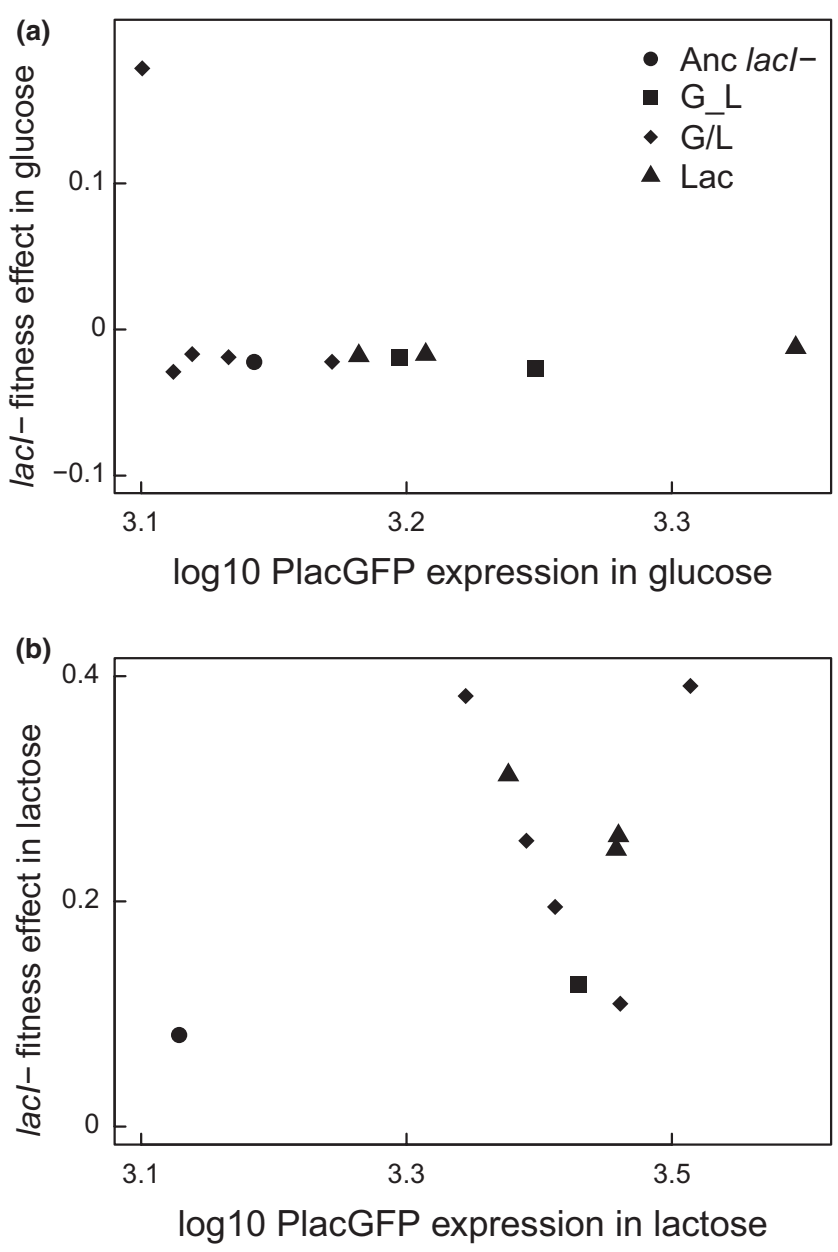

FIGURE 5 Relationship between lac expression and laclfitness effect. Symbols indicate the mean fitness effect and lacreporter expression of the ancestor and evolved clones as indicated in panel a. (a) Expression and fitness estimates measured in the glucose environment. (b) Expression and fitness estimates measured in the lactose environment

for loss of other peptidases involved in periplasm protein recycling, suggesting a possibility of affecting LacY function.

An alternative mechanism of lacl- compensation is a change in lacl regulation. This could occur in either of two ways. First, the 4 bp insertion frameshift mutation in lacl- of G/L4 results in a truncated 204 residue Lacl repressor. Although nonfunctional at the lac operator, it is possible that the truncated Lacl, which includes the DNA binding domain, interacts with another region in the genome to cause some beneficial effect that is lost when the ancestral lacl was restored (Platt et al., 1973). Second, an operator site for a different gene may have evolved to provide a benefit in the absence of Lacl. The Lacl repressor has similarity to other repressors in E. coli (Weickert \& Adhya, 1992), and studies have shown that as few as two mutations are needed to increase the affinity of Lacl to another operator (Daber \& Lewis, 2009; Lehming et al., 1987, 1990; Salinas et al., 2005). We note, however, that the sequenced $G / L 4$ clone does not have mutations in recognized lacl family operator sites, so a change in sites bound by Lacl is not predicted (Table S1).
In summary, compensation was rare and did not occur based on specific fluctuations of glucose and lactose. In the clone in which compensation of lacl- did occur, lac operon expression was reduced but not more so than other strains that had a similar cost in glucose compared to the ancestor. This indicates that costs of constitutive expression were overcome by epistatic interactions with other mutations in the evolved background and that the reduction in cost was not due solely to a reduction in expression. Future studies will examine potential long-term trade-offs of compensation and what makes compensation rare.

\section{ACKNOWLEDGMENTS}

This work was supported by grant DEB-1253650 awarded to T.F.C. by the National Science Foundation and by grant 19-MAU-082 awarded to T.F.C. by the Royal Society of New Zealand Marsden fund.

\section{CONFLICT OF INTEREST}

The authors declare no conflict of interest.

\section{AUTHOR CONTRIBUTIONS}

Kelly N. Phillips: Conceptualization (equal); Data curation (equal); Formal analysis (equal); Investigation (equal); Methodology (equal); Software (lead); Writing-original draft (equal); Writing-review \& editing (equal). Tim F. Cooper: Conceptualization (equal); Data curation (equal); Formal analysis (equal); Funding acquisition (equal); Methodology (equal); Project administration (equal); Software (supporting); Visualization (equal); Writing-original draft (equal); Writingreview \& editing (equal).

\section{DATA AVAILABILITY STATEMENT}

T.F.C will make the strains constructed in this study available to qualified recipients following completion of an institutional material transfer agreement. The results of competition experiments, summary input data, and analysis scripts that pertain to the experiments and analyses reported in this paper have been deposited at DOI https://doi.org/10.5061/dryad.8sf7m0cp2.

\section{ORCID}

Tim F. Cooper iD https://orcid.org/0000-0001-5038-9366

\section{REFERENCES}

Bailey, S. F., \& Kassen, R. (2012). Spatial structure of ecological opportunity drives adaptation in a bacterium. American Naturalist, 180, 270283. https://doi.org/10.1086/666609

Baird, L., Steinsiek, B. L., Raina, S., \& Georgopoulos, C. (1991). Identification of the Escherichia coli sohB gene, a multicopy suppressor of the HtrA (DegP) null phenotype. Journal of Bacteriology, 173, 5763-5770.

Bennett, A. F., \& Lenski, R. E. (2007). An experimental test of evolutionary trade-offs during temperature adaptation. Proceedings of the National Academy of Sciences of the United States of America, 104, S8649-8654. https://doi.org/10.1073/pnas.0702117104

Björkman, J., Hughes, D., \& Andersson, D. I. (1998). Virulence of antibioticresistant Salmonella typhimurium. Proceedings of the National Academy 
of Sciences of the United States of America, 95, 3949-3953. https://doi. org/10.1073/pnas.95.7.3949

Björkman, J., Nagaev, I., Berg, O. G., Hughes, D., \& Andersson, D. I. (2000). Effects of environment on compensatory mutations to ameliorate costs of antibiotic resistance. Science, 287, 1479-1482. https://doi.org/10.1126/science.287.5457.1479

Blank, D., Wolf, L., Ackermann, M., \& Silander, O. K. (2014). The predictability of molecular evolution during functional innovation. Proceedings of the National Academy of Sciences of the United States of America, 111, 3044-3049. https://doi.org/10.1073/pnas.13187 97111

Blount, Z. D., Barrick, J. E., Davidson, C. J., \& Lenski, R. E. (2012). Genomic analysis of a key innovation in an experimental Escherichia coli population. Nature, 489, 513-518. https://doi.org/10.1038/nature11514

Blount, Z. D., Borland, C. Z., \& Lenski, R. E. (2008). Historical contingency and the evolution of a key innovation in an experimental population of Escherichia coli. Proceedings of the National Academy of Sciences of the United States of America, 105, 7899-7906. https://doi. org/10.1073/pnas.0803151105

Bono, L. M., Smith, L. B., Pfennig, D. W., \& Burch, C. L. (2017). The emergence of performance trade-offs during local adaptation: Insights from experimental evolution. Molecular Ecology, 26, 1720-1733. https://doi.org/10.1111/mec.13979

Brandis, G., Wrande, M., Liljas, L., \& Hughes, D. (2012). Fitnesscompensatory mutations in rifampicin-resistant RNA polymerase. Molecular Microbiology, 85, 142-151. https://doi. org/10.1111/j.1365-2958.2012.08099.x

Buckling, A., Brockhurst, M. A., Travisano, M., \& Rainey, P. B. (2007). Experimental adaptation to high and low quality environments under different scales of temporal variation. Journal of Evolutionary Biology, 20, 296-300. https://doi.org/10.1111/j.1420-9101.2006.01195.x

Buckling, A., Kassen, R., Bell, G., \& Rainey, P. B. (2000). Disturbance and diversity in experimental microcosms. Nature, 408, 961-964. https:// doi.org/10.1038/35050080

Choi, K.-H., Gaynor, J. B., White, K. G., Lopez, C., Bosio, C. M., KarkhoffSchweizer, R. R., \& Schweizer, H. P. (2005). A Tn7-based broad-range bacterial cloning and expression system. Nature Methods, 2, 443448. https://doi.org/10.1038/nmeth765

Cooper, T. F., \& Lenski, R. E. (2010). Experimental evolution with E. coli in diverse resource environments. I. Fluctuating environments promote divergence of replicate populations. BMC Evolutionary Biology, 10, 11-10. https://doi.org/10.1186/1471-2148-10-11

Cooper, T. F., Rozen, D. E., \& Lenski, R. E. (2003). Parallel changes in gene expression after 20,000 generations of evolution in Escherichia coli. Proceedings of the National Academy of Sciences of the United States of America, 100, 1072-1077.

Covert, A. W., Lenski, R. E., Wilke, C. O., \& Ofria, C. (2013). Experiments on the role of deleterious mutations as stepping stones in adaptive evolution. Proceedings of the National Academy of Sciences of the United States of America, 110, E3171-E3178. https://doi.org/10.1073/ pnas.1313424110

Daber, R., \& Lewis, M. (2009). A novel molecular switch. Journal of Molecular Biology, 391, 661-670. https://doi.org/10.1016/j. jmb.2009.06.039

Deatherage, D. E., \& Barrick, J. E. (2014). Identification of mutations in laboratory-evolved microbes from next-generation sequencing data using breseq. Methods in Molecular Biology, 1151, 165-188.

Dekel, E., \& Alon, U. (2005). Optimality and evolutionary tuning of the expression level of a protein. Nature, 436, 588-592. https://doi. org/10.1038/nature03842

Eames, M., \& Kortemme, T. (2012). Cost-benefit tradeoffs in engineered lac operons. Science, 336, 911-915. https://doi.org/10.1126/scien ce.1219083

Ferenci, T. (1996). Adaptation to life at micromolar nutrient levels: The regulation of Escherichia coli glucose transport by endoinduction and cAMP. FEMS Microbiology Reviews, 18, 301-317.
Ferrières, L., Hémery, G., Nham, T., Guérout, A.-M., Mazel, D., Beloin, C., \& Ghigo, J. M. (2010). Silent mischief: Bacteriophage Mu insertions contaminate products of Escherichia coli random mutagenesis performed using suicidal transposon delivery plasmids mobilized by broad-host-range RP4 conjugative machinery. Journal of Bacteriology, 192, 6418-6427.

Filteau, M., Hamel, V., Pouliot, M.-C., Gagnon-Arsenault, I., Dube, A. K., \& Landry, C. R. (2015). Evolutionary rescue by compensatory mutations is constrained by genomic and environmental backgrounds. Molecular Systems Biology, 11, 832.

Gerrish, P., \& Lenski, R. E. (1998). The fate of competing beneficial mutations in an asexual population. Genetica, 102-103, 127-144.

Gong, L. I., Suchard, M. A., \& Bloom, J. D. (2013). Stability-mediated epistasis constrains the evolution of an influenza protein. eLife, 2, e00631.

Gram, C. D., \& Brooker, R. J. (1992). An analysis of the side chain requirement at position 177 within the lactose permease which confers the ability to recognize maltose. Journal of Biological Chemistry, 267, 3841-3846. https://doi.org/10.1016/S0021-9258(19)50602-0

Harrison, E., Guymer, D., Spiers, A. J., Paterson, S., \& Brockhurst, M. A. (2015). Parallel compensatory evolution stabilizes plasmids across the parasitism-mutualism continuum. Current Biology, 25, 20342039. https://doi.org/10.1016/j.cub.2015.06.024

Jahreis, K., Pimentel-Schmitt, E. F., Brueckner, R., \& Titgemeyer, F. (2008). Ins and outs of glucose transport systems in eubacteria. FEMS Microbiology Reviews, 32, 891-907. https://doi. org/10.1111/j.1574-6976.2008.00125.x

Jarvik, J., \& Botstein, D. (1975). Conditional lethal mutations that suppress genetic defects in morphogenesis by altering structural proteins. Proceedings of the National Academy of Sciences of the United States of America, 72, 2738-2742. https://doi.org/10.1073/pnas.72.7.2738

Jasmin, J.-N., \& Kassen, R. (2007). Evolution of a single niche specialist in variable environments. Proceedings of the Royal Society of London, Series B: Biological Sciences, 274, 2761-2767. https://doi.org/10.1098/ rspb.2007.0936

Kacar, B., Ge, X., Sanyal, S., \& Gaucher, E. A. (2017). Experimental evolution of Escherichia coli harboring an ancient translation protein. Journal of Molecular Evolution, 84, 69-84. https://doi.org/10.1007/ s00239-017-9781-0

Kassen, R., \& Bell, G. (1998). Experimental evolution in Chlamydomonas. IV. Selection in environments that vary through time at different scales. Heredity, 80, 732-741. https://doi. org/10.1046/j.1365-2540.1998.00329.x

King, S. C., \& Wilson, T. H. (1990). Identification of valine 177 as a mutation altering specificity for transport of sugars by the Escherichia coli lactose carrier. Enhanced specificity for sucrose and maltose. Journal of Biological Chemistry, 265, 9638-9644. https://doi.org/10.1016/ S0021-9258(19)38717-4

Knöppel, A., Nasvall, J., \& Andersson, D. I. (2016). Compensating the fitness costs of synonymous mutations. Molecular Biology and Evolution, 33, 1461-1477. https://doi.org/10.1093/molbev/msw028

Kurlandzka, A., Rosenzweig, R. F., \& Adams, J. (1991). Identification of adaptive changes in an evolving population of Escherichia coli: The role of changes with regulatory and highly pleiotropic effects. Molecular Biology and Evolution, 8, 261-281.

Lee, M.-C., \& Marx, C. J. (2012). Repeated, selection-driven genome reduction of accessory genes in experimental populations. PLoS Genetics, 8, e1002651.

Lehming, N., Sartorius, J., Kisters-Woike, B., von Wilcken-Bergmann, B., \& Müller-Hill, B. (1990). Mutant lac repressors with new specificities hint at rules for protein-DNA recognition. EMBO Journal, 9, 615-621. https://doi.org/10.1002/j.1460-2075.1990.tb08153.x

Lehming, N., Sartorius, J., Niemöller, M., Genenger, G., von WilckenBergmannand, B., \& Müller-Hill, B. (1987). The interaction of the recognition helix of lac repressor with lac operator. EMBO Journal, 6 , 3145-3153. https://doi.org/10.1002/j.1460-2075.1987.tb02625.x 
Lenski, R. E. (1988). Experimental studies of pleiotropy and epistasis in Escherichia coli. II. Compensation for maldaptive effects associated with resistance to virus T4. Evolution, 42, 433-440.

Lenski, R. E., Rose, M. R., Simpson, S. C., \& Tadler, S. C. (1991). Long-term experimental evolution in Escherichia coli. I. Adaptation and divergence during 2,000 generations. American Naturalist, 138, 13151341. https://doi.org/10.1086/285289

Leon, D., D'Alton, S., Quandt, E. M., \& Barrick, J. E. (2018). Innovation in an $E$. coli evolution experiment is contingent on maintaining adaptive potential until competition subsides. PLoS Genetics, 14, e1007348. https://doi.org/10.1371/journal.pgen.1007348

Levin, B. R., Perrot, V., \& Walker, N. (2000). Compensatory mutations, antibiotic resistance and the population genetics of adaptive evolution in bacteria. Genetics, 154, 985-997.

Lunzer, M., Golding, G. B., \& Dean, A. M. (2010). Pervasive cryptic epistasis in molecular evolution. PLoS Genetics, 6, e1001162. https://doi. org/10.1371/journal.pgen.1001162

MacLean, R. C., Bell, G., \& Rainey, P. B. (2004). The evolution of a pleiotropic fitness tradeoff in Pseudomonas fluorescens. Proceedings of the National Academy of Sciences of the United States of America, 101, 8072-8077. https://doi.org/10.1073/pnas.0307195101

Maisnier-Patin, S., Berg, O. G., Liljas, L., \& Andersson, D. I. (2002). Compensatory adaptation to the deleterious effect of antibiotic resistance in Salmonella typhimurium. Molecular Microbiology, 46, 355-366.

Manson, M. D. (2000). Allele-specific suppression as a tool to study protein-protein interactions in bacteria. Methods, 20, 18-34. https:// doi.org/10.1006/meth.1999.0902

Markiewicz, P., Kleina, L. G., Cruz, C., Ehret, S., \& Miller, J. H. (1994). Genetic studies of the lac repressor. XIV. Analysis of 4000 altered Escherichia coli lac repressors reveals essential and non-essential residues, as well as "spacers" which do not require a specific sequence. Journal of Molecular Biology, 240, 421-433.

Martin, G., \& Lenormand, T. (2015). The fitness effect of mutations across environments: Fisher's geometrical model with multiple optima. Evolution, 69, 1433-1447. https://doi.org/10.1111/evo.12671

McGee, L. W., Sackman, A. M., Morrison, A. J., Pierce, J., Anisman, J., \& Rokyta, D. R. (2015). Synergistic pleiotropy overrides the costs of complexity in viral adaptation. Genetics, 202, 285-295. https://doi. org/10.1534/genetics.115.181628

Melnyk, A. H., McCloskey, N., Hinz, A. J., Dettman, J., \& Kassen, R. (2017). Evolution of cost-free resistance under fluctuating drug selection in Pseudomonas aeruginosa. mSphere, 2, e00158-17.

Moore, F., Rozen, D. E., \& Lenski, R. E. (2000). Pervasive compensatory adaptation in Escherichia coli. Proceedings of the Royal Society of London, Series B: Biological Sciences, 267, 515-522.

Moura de Sousa, J., Balbontín, R., Durão, P., \& Gordo, I. (2017). Multidrug-resistant bacteria compensate for the epistasis between resistances. PLoS Biology, 15, e2001741. https://doi.org/10.1371/ journal.pbio.2001741

Nagaev, I., Björkman, J., Andersson, D. I., \& Hughes, D. (2001). Biological cost and compensatory evolution in fusidic acid-resistant Staphylococcus aureus. Molecular Microbiology, 40, 433-439. https:// doi.org/10.1046/j.1365-2958.2001.02389.x

Nilsson, A. I., Berg, O. G., Aspevall, O., Kahlmeter, G., \& Andersson, D. I. (2003). Biological costs and mechanisms of fosfomycin resistance in Escherichia coli. Antimicrobial Agents and Chemotherapy, 47, 2850-2858

Philippe, N., Alcaraz, J.-P., Coursange, E., Geiselmann, J., \& Schneider, D. (2004). Improvement of pCVD442, a suicide plasmid for gene allele exchange in bacteria. Plasmid, 51, 246-255.

Phillips, K. N., Castillo, G., Wünsche, A., \& Cooper, T. F. (2016). Adaptation of Escherichia coli to glucose promotes evolvability in lactose. Evolution, 70, 465-470.
Platt, T., Files, J. G., \& Weber, K. (1973). Lac repressor: Specific proteolytic destruction of the $\mathrm{NH}_{2}$-terminal region and loss of the deoxyribonucleic acid-binding activity. Journal of Biological Chemistry, 248, 110-121. https://doi.org/10.1016/S0021-9258(19)44452-9

Ponmani, T., \& Munavar, M. H. (2014). G673 could be a novel mutational hot spot for intragenic suppressors of pheS5 lesion in Escherichia coli. Microbiology, 3, 369-382.

Poon, A., \& Chao, L. (2005). The rate of compensatory mutation in the DNA bacteriophage $\varnothing$ X174. Genetics, 170, 989-999.

Poon, A., \& Chao, L. (2006). Functional origins of fitness effectsizes of compensatory mutations in the DNA bacteriophage $\varnothing \times 174 . \quad E v o l u t i o n, 60,2032-2043$. https://doi.org/10.1111/ j.0014-3820.2006.tb01841.x

Poon, A., Davis, B. H., \& Chao, L. (2005). The coupon collector and the suppressor mutation: Estimating the number of compensatory mutations by maximum likelihood. Genetics, 170, 1323-1332. https://doi. org/10.1534/genetics.104.037259

Postma, P. W., Lengeler, J. W., \& Jacobson, G. R. (1993). Phosphoenolpyruvate:Carbohydrate phosphotransferase systems of bacteria. Microbiological Reviews, 57, 543-594. https://doi. org/10.1128/mr.57.3.543-594.1993

Quan, S., Ray, J. C. J., Kwota, Z., Duong, T., Balazsi, G., Cooper, T. F., \& Monds, R. D. (2012). Adaptive evolution of the lactose utilization network in experimentally evolved populations of Escherichia coli. PLoS Genetics, 8, e1002444. https://doi.org/10.1371/journ al.pgen.1002444

Quandt, E. M., Gollihar, J., Blount, Z. D., Ellington, A. D., Georgiou, G., \& Barrick, J. E. (2015). Fine-tuning citrate synthase flux potentiates and refines metabolic innovation in the Lenski evolution experiment. eLife, 4, e09696. https://doi.org/10.7554/eLife.09696

R Core Team (2018). R: A language and environment for statistical computing. R Foundation for Statistical Computing. https://www.R-proje ct.org/

Roemhild, R., Barbosa, C., Beardmore, R. E., Jansen, G., \& Schulenburg, H. (2015). Temporal variation in antibiotic environments slows down resistance evolution in pathogenic Pseudomonas aeruginosa. Evolutionary Applications, 8, 945-955.

Rosenzweig, R. F., Sharp, R. R., Treves, D. S., \& Adams, J. (1994) Microbial evolution in a simple unstructured environment: Genetic differentiation in Escherichia coli. Genetics, 137, 903-917. https://doi. org/10.1093/genetics/137.4.903

Rozen, D. E., McGee, L., Levin, B. R., \& Klugman, K. P. (2007). Fitness costs of fluoroquinolone resistance in Streptococcus pneumoniae. Antimicrobial Agents and Chemotherapy, 51, 412-416.

Sahin-Tóth, M., Lawrence, M. C., Nishio, T., \& Kaback, H. R. (2001). The $\mathrm{C}-4$ hydroxyl group of galactopyranosides is the major determinant for ligand recognition by the lactose permease of Escherichia coli. Biochemistry, 40, 13015-13019.

Salinas, R. K., Folkers, G. E., Bonvin, A. M. J. J., Das, D., Boelens, R., \& Kaptein, R. (2005). Altered specificity in DNA binding by the lac repressor: A mutant lac headpiece that mimics the gal repressor. ChemBioChem, 6, 1628-1637. https://doi.org/10.1002/cbic.20050 0049

Satterwhite, R. S., \& Cooper, T. F. (2015). Constraints on adaptation of Escherichia coli to mixed-resource environments increase over time. Evolution, 69, 2067-2078.

Schick, A., Bailey, S. F., \& Kassen, R. (2015). Evolution of fitness tradeoffs in locally adapted populations of Pseudomonas fluorescens. American Naturalist, 186, S48-S59.

Shah, P., McCandlish, D. M., \& Plotkin, J. B. (2015). Contingency and entrenchment in protein evolution under purifying selection. Proceedings of the National Academy of Sciences of the United States of America, 112, E3226-E3235. https://doi.org/10.1073/pnas.14129 33112 
Stoebel, D. M., Dean, A. M., \& Dykhuizen, D. E. (2008). The cost of expression of Escherichia coli lac operon proteins is in the process, not in the products. Genetics, 178, 1653-1660.

Szamecz, B., Boross, G., Kalapis, D., Kovács, K., Fekete, G., Farkas, Z., Lázár, V., Hrtyan, M., Kemmeren, P., Groot Koerkamp, M. J. A., Rutkai, E., Holstege, F. C. P., Papp, B., \& Pál, C. (2014). The genomic landscape of compensatory evolution. PLoS Biology, 12, e1001935. https://doi.org/10.1371/journal.pbio.1001935

Tenaillon, O., Barrick, J. E., Ribeck, N., Deatherage, D. E., Blanchard, J. L., Dasgupta, A., Wu, G. C., Wielgoss, S., Cruveiller, S., Médigue, C., Schneider, D., \& Lenski, R. E. (2016). Tempo and mode of genome evolution in a 50,000-generation experiment. Nature, 536, 165-170.

Turner, P. E., \& Elena, S. F. (2000). Cost of host radiation in an RNA virus. Genetics, 156, 1465-1470. https://doi.org/10.1093/genet ics/156.4.1465

van Leeuwen, J., Pons, C., Mellor, J. C., Yamaguchi, T. N., Friesen, H., Koschwanez, J., Uaj, M. M., Pechlaner, M., Takar, M., Uaj, M., VanderSluis, B., Andrusiak, K., Bansal, P., Baryshnikova, A., Boone, C. E., Cao, J., Cote, A., Gebbia, M., Horecka, G., ... Boone, C. (2016). Exploring genetic suppression interactions on a global scale. Science, 354, aag0839-1-aag0839-11. https://doi.org/10.1126/scien ce.aag0839

Weickert, M. J., \& Adhya, S. (1992). A family of bacterial regulators homologous to Gal and Lac repressors. Journal of Biological Chemistry, 267, 15869-15874. https://doi.org/10.1016/S0021-9258(19)49615 $-4$

Wielgoss, S., Bergmiller, T., Bischofberger, A. M., \& Hall, A. R. (2016). Adaptation to parasites and costs of parasite resistance in mutator and nonmutator bacteria. Molecular Biology and Evolution, 33, 770782. https://doi.org/10.1093/molbev/msv270

Wood, M. P., Cole, A. L., Ruchala, P., Waring, A. J., Rohan, L. C., Marx, P., Tarwater, P. M., Gupta, P., \& Cole, A. M. (2013). A compensatory mutation provides resistance to disparate HIV fusion inhibitor peptides and enhances membrane fusion. PLoS One, 8, e55478. https:// doi.org/10.1371/journal.pone.0055478

Woods, R., Schneider, D., Winkworth, C. L., Riley, M. A., \& Lenski, R. E. (2006). Tests of parallel molecular evolution in a long-term experiment with Escherichia coli. Proceedings of the National Academy of Sciences of the United States of America, 103, 9107-9112. https://doi. org/10.1073/pnas.0602917103

Zee, P. C., Mendes-Soares, H., Yu, Y.-T., Kraemer, S. A., Keller, H., Ossowski, S., Schneeberger, K., \& Velicer, G. J. (2014). A shift from magnitude to sign epistasis during adaptive evolution of a bacterial social trait. Evolution, 68, 2701-2708. https://doi.org/10.1111/ evo.12467

\section{SUPPORTING INFORMATION}

Additional supporting information may be found online in the Supporting Information section.

How to cite this article: Phillips, K. N., \& Cooper, T. F. (2021).

The cost of evolved constitutive lac gene expression is usually, but not always, maintained during evolution of generalist populations. Ecology and Evolution, 11, 1249712507. https://doi.org/10.1002/ece3.7994 